\title{
Treatable Mechanisms in Asthma
}

\author{
Mario Cazzola ${ }^{1}\left[\right.$ D $\cdot$ Josuel Ora ${ }^{2} \cdot$ Francesco Cavalli $^{2} \cdot$ Paola Rogliani ${ }^{1,2} \cdot$ Maria Gabriella Matera $^{3}$
}

Accepted: 12 January 2021

(c) The Author(s) 2021

\begin{abstract}
Asthma is a heterogeneous condition, but firm identification of heterogeneity-focused treatments is still lacking. Dividing patients into subgroups of asthma pheno-/endotypes based on combined clinical and cellular biological characteristics and linking them to targeted treatments could be a potentially useful approach to personalize therapy for better outcomes. Nonetheless, there are still many problems related to the identification and validation of asthma phenotypes and endotypes. Alternatively, a precision-medicine strategy for the management of patients with airways disease that is free from the traditional diagnostic labels and based on identifying "treatable traits" in each patient might be preferable. However, it would represent a quite unsophisticated approach because the definition of a treatable trait is too imprecise. In fact, there is still no understanding of the mechanisms underlying treatable traits that allow directing any targeted therapies against any particular treatable trait. Fortunately, in-depth identification of underlying molecular pathways to guide targeted treatment in individual patients is in progress thanks to the improvement in big data management obtained from '-omic' sciences that is greatly increasing knowledge concerning asthma.
\end{abstract}

\section{Asthma is a Heterogeneous Disorder}

In 2007, asthma was defined as "a common chronic disorder of the airways that is complex and characterized by variable and recurring symptoms, airflow obstruction, bronchial hyperresponsiveness, and an underlying inflammation" [1]. However, appropriately Lötvall and colleagues pointed out that when examining large cohorts of patients with asthma, all of these features are often not present concurrently, or one or two may predominate [2]. What is becoming increasingly accepted is the concept expressed several years ago by Sally Wenzel that asthma is not a single disease, but rather a complex of multiple, separate and different syndromes that often overlap [3]. In effect, 'asthma' is an umbrella term that is used to give a simplified definition of grouped clinical and physiological characteristics [4].

In its last version, the GINA (Global Initiative for Asthma) report, while maintaining the concept that asthma

Mario Cazzola

mario.cazzola@uniroma2.it

1 Department of Experimental Medicine, University of Rome "Tor Vergata", Rome, Italy

2 Respiratory Diseases Unit, "Tor Vergata” University Hospital, Rome, Italy

3 Department of Experimental Medicine, University of Campania “Luigi Vanvitelli”, Naples, Italy

\section{Key Points}

Precision medicine is now becoming central in the treatment of airways diseases, including asthma, which is an umbrella term that is used to give a simplified definition of grouped clinical and physiological characteristics (phenotypes) and distinct mechanistic pathways (endotypes).

Asthma exhibits high heterogeneity across patient populations with few (FeNO and blood eosinophils) established biomarkers, but the awareness of this heterogeneity has not yet been accompanied by firm identification of heterogeneity-focused treatments.

The 'treatable traits' approach is an interesting attempt to introduce precision medicine in asthma, and the identification of treatable traits could help optimize treatment in this heterogeneous disorder.

It is widely agreed that additional knowledge about the mechanisms underlying these treatable traits is needed to correctly guide targeted-treatment based on identified traits in the individual patient. A clever and accurate application of the '-omic' sciences can allow the replacement of phenotypes with the current emerging concept of 'treatable mechanisms'. 
is a disease, has defined it as "a heterogeneous disease, usually characterized by chronic airway inflammation. It is defined by the history of respiratory symptoms such as wheeze, shortness of breath, chest tightness and cough that vary over time and in intensity, together with variable expiratory airflow limitation" [5]. The use of the adjective "heterogeneous" certainly represents a significant step forward compared to the definition of 2007. In fact, asthma is a complex condition in which a dysregulated immune response, chronic inflammation, tissue remodelling and hyperresponsiveness in the affected tissues are combined in a completely heterogeneous but dynamic manner [6].

However, although asthma has been defined as a heterogeneous condition, this has not been accompanied by the firm identification of heterogeneity-focused treatments. Treatments that are currently used certainly improve symptoms in the long term, but do not significantly affect intraindividual and even temporal variability of the various features that characterize asthma. This is certainly a major limitation of the existing asthma therapies, especially considering that the methodology of modern medicine is to offer the patient a proactive and personalized approach that combines precision diagnosis and personalized treatment [7]. In recent years, considerable efforts have been made to understand the various clinical forms of asthma and their pathophysiology, especially in order to develop a more targeted therapeutic approach to the individual patient's condition [8]. Unfortunately, the goal of providing personalized care to each patient with asthma is still difficult to achieve. There is definitely a need to switch to personalized treatment in asthma, although unfortunately a specific therapy cannot always be identified.

This review addresses the question of personalized medicine for asthma care with a specific focus on the treatable traits approach, and the promise of the application of the '-omic' sciences to advance more personalized care for the asthmatic patient. We begin by discussing what 'precision medicine' means. We then consider the pheno-/endotyping approach. Afterwards, we briefly review the possibility of using the treatable traits approach in asthma, how effective it actually is, and where there is room for improvement. We conclude with what we need to get from the '-omic' sciences to improve our ability to effectively use precision medicine in asthma in order to predict an asthma drug-treatment response.

\section{Precision Medicine}

The fundamental idea behind a personalized approach is coupling established clinical-pathological indexes with state-of-the-art molecular profiling to generate diagnostic, prognostic and therapeutic strategies specifically tailored to each patient's needs. The term 'precision medicine' stems from this concept [9]. Precision medicine links the right patient to the right treatment. It should ensure that patients get the right treatment at the right dose at the right time, with minimum harmful consequences and maximum efficacy [10].

In recent years, there has been and continues to be a flourish of information that is leading to a better understanding of what is called 'precision medicine'. Obviously, the development of precision medicine brought about its own taxonomy [6]. Apparently abstruse terms such as phenotype, endotype, biomarker, theratype, regiotype, inflammasome endotype, barrier endotype, stratified medicine and biomarkers, enrichment, and prognostic enrichment are becoming progressively better understood not only by researchers but also by clinicians [6].

This means there is a strong need to classify individuals into sub-populations that differ in their susceptibility to a particular disease or in response to a specific treatment [9].

\section{Defining Asthma Phenotypes}

Several clinical features can occur with varying percentages in each individual patient with asthma, and the overlap of separate and different characteristics makes it difficult to distinctly classify affected patients [3]. It has been suggested that these characteristics could identify syndromes, phenotypes or even multiple diseases rather than a single disease [4].

Usually, a phenotype is considered the physical appearance or biochemical characteristic resulting from an interaction between its genotype and the environment. It describes 'observable characteristics' such as clinical, physiological, morphologic and biochemical characteristics as well as the response to different treatments, with no direct relationship to a disease process including physiology, triggers and inflammatory parameters [2].

Identification of the phenotype of a patient's asthma is considered important because it can assist in management [3]. In fact, it seems possible to identify different asthmatic phenotypes considering the clinical features (lung function, symptoms and exacerbations) indicating the severity of asthma, its inflammatory characteristics and the overlap of these clinical features and inflammatory characteristics, although these phenotypes have not yet been fully characterized [4].

The initial approach that had been used to define asthma phenotypes classified them into three categories: phenotypes defined by clinical or physiological criteria, which included those defined by level of severity (from mild to severe), the frequency of exacerbations, the presence of chronic airflow restriction, and the age of asthma onset; phenotypes related to environmental triggers, including exercise, environmental 
allergens, occupational allergens and irritants, drugs (such as aspirin), and menses; and phenotypes defined by their pathobiology, specifically the presence or absence of particular inflammatory cell types, for example eosinophils or neutrophils [3]. However, it is currently unclear whether asthma involves a collection of different conditions, or is a single condition with multiple mechanisms and phenotypes, or what the underlying phenotypes may be [11].

The majority of, but not all, clinical asthma cases are assumed to be the net result of excessive inflammation driven by an aberrant T-helper-2 $\left(\mathrm{T}_{\mathrm{H}} 2\right)$ process that is strongly linked to atopy and allergy, type I hypersensitivity reactions, eosinophilic inflammation, remodelled airways, and then functional derangement, which, in turn, causes symptoms and response to corticosteroids [4]. Therefore, an oversimplification of phenotyping might be to divide asthma into two major subtypes-Type-2 high and Type-2 low [12]. Type- 2 high or T2 or T2 high or T-helper $2\left(\mathrm{~T}_{\mathrm{H}} 2\right)$-high exhibits increased eosinophilia and other signs of Type-2 airway inflammation, including increased numbers of airway mast cells subgroups, compared with the normal reference range of healthy controls. Type- 2 low or non-T2 or T2 low or $\mathrm{T}_{\mathrm{H}} 2$ low is characterized by levels of Type 2 inflammation in the airways comparable to those found in healthy subjects [12]. It has been reported that the prevalence of Type-2 high patients is $89 \%$, while that of Type- 2 low patients is $11 \%$ [13]. However, in another study, at initial assessment $71 \%$ of an adult population of patients with a diagnosis of difficult asthma were Type-2 high, $18.5 \%$ were Type- 2 low and $10.5 \%$ were Type- 2 intermediate, but only $8 \%$ of these asthmatics appeared persistently Type- 2 low once their inhaled corticosteroid (ICS) or oral corticosteroid dose was optimised [14].

Type- 2 high asthma can in turn be divided into an earlyand late-onset disorder, although severity varies greatly regardless of early or late onset of asthma [4]. Generally, early-onset allergic asthma is mild, but it can occur with increased severity in the presence of an increasing complexity of immune processes. Type- 2 high asthma that appears later in life in the absence of traditional allergic elements is frequently severe.

There are also forms of Type-2 low asthma with very late onset that are related to $\mathrm{T}_{\mathrm{H}} 17$ and $\mathrm{T}_{\mathrm{H}} 1$, such as those that are associated with obesity or/and smoking, neutrophilic asthma and also low-inflammatory asthma [4]. Essentially, many asthmatics do not show active Type-2 airway inflammation even in the presence of smooth muscle dysfunction [12]. This feature is very important because it suggests the lack of a link between Type-2 inflammation and the presence of excessive smooth muscle tone and bronchial hyperreactivity, which characterise asthma. Instead, it would seem that Type- 2 inflammation plays a central role in altering asthma control and increasing susceptibility to exacerbations [12].
Unfortunately, the understanding of Type-2 low asthma is still poor and therefore it is appropriate to wonder whether it is a distinct molecular phenotype or Type-2 high asthma in remission, and even if it presents temporal stability [15].

Type- 2 high asthma is characterized by an effective treatment response with ICSs, which are the anti-inflammatory drugs of choice for asthma, while Type-2 low asthma is not [16].

Beginning with the axiom that a single clinical, biological or physiological characteristic is not sufficient to define a phenotype but there is a need to integrate more characteristics, many different approaches to phenotyping have been proposed, with a focus on clinical/physiological characteristics, triggering factors and inflammatory components [17].

Clustering patients defined by clinical and physiological characteristics and different pathobiological pathways using unbiased cluster analytic methods allows for the identification of different phenotypes when examining patient cohorts that are representative of the full spectrum of asthma severity [8]. The clusterisation of the European Unbiased Biomarkers for the Prediction of Respiratory Diseases Outcomes (U-BIOPRED) cohort of adults with moderate-tosevere asthma using eight prespecified clinic-physiologic variables led to the identification of four reproducible and stable clusters associated with different pathobiological pathways [18]. Cluster 1 consisted of patients with wellcontrolled moderate-to-severe asthma, with normal $\mathrm{FEV}_{1}$ (forced expiratory volume in $1 \mathrm{~s}$ ) and low sputum eosinophilia, whereas cluster 2 comprised a group of overweight to obese patients with late-onset severe asthma, a history of smoking, relatively poor control, chronic airflow obstruction and the highest sputum and blood eosinophilia. Cluster 3 was similar to cluster 2 in terms of chronic airflow obstruction but comprised patients who were non-smokers and less overweight, had poorer lung function, and had a higher proportion of atopic participants. Cluster 4 was predominantly composed of obese female patients with uncontrolled severe asthma and increased exacerbations but with normal lung function.

In addition, the clusterisation of the Airway Disease Endotyping for Personalized Therapeutics (ADEPT) cohort, using distinct clinical and biomarker profiles, allowed for identification of four reproduced clusters with good longitudinal stability [19]. Cluster 1 included patients with mild, good lung function, early onset and a low inflammatory, predominantly Type- 2 , asthma. Patients in cluster 2 had a moderate, hyperresponsive, eosinophilic form of asthma, with moderate control, mild airflow obstruction and predominant Type-2 inflammation. Cluster 3 consisted of patients with mixed severity, predominantly fixed obstructive, Type- 2 low inflammation (non-eosinophilic and neutrophilic) and moderately controlled asthma, whereas cluster 4 patients presented with severe uncontrolled, severe reversible 
obstruction, mixed granulocytic asthma, and moderate Type-2 inflammation.

Recently, Kaur and Chupp have observed that there is repeated evidence suggesting the existence of approximately four main phenotypes of asthma, regardless of the differences in the cohorts examined, the disease characteristics used in each clustering analysis, the computational approaches, and the number of clusters identified in the single study [20]. According to their view, patients with early-onset mild allergic asthma, with early-onset allergic moderate-to-severe remodelled asthma, with late-onset nonallergic eosinophilic asthma, or with late-onset non-eosinophilic non-allergic asthma are commonly encountered. In the heterogeneity of asthma, the age of onset, lung function and presence or absence of atopy are the key discriminatory factors, with eosinophils being a key biomarker and effector cells in asthmatic patients.

Understanding the phenotypes of asthma in older adults is very important because age of asthma onset within the general adult asthma population can affect clinical and inflammatory variables [21]. In fact, severe adult-onset asthma may be a distinct phenotype compared to milder forms of adult-onset asthma. It is associated with a greater proportion of non-atopics, worse nasal symptoms, greater severity but often less perceived airway obstruction, higher levels of inflammatory biomarkers such as fractional exhaled nitric oxide (FeNO), blood neutrophils and sputum eosinophils, and frequent co-morbidities [22]. In addition, sex-specific asthma phenotypes have also been found [23]. In females, atopy with an eosinophil-predominant phenotype and obesity with neutrophil-predominant inflammation phenotype have been identified. Two distinct asthma phenotypes were found in current (slightly high blood neutrophils and low blood eosinophils) and ex-smokers (eosinophil-predominant or mixed inflammatory pattern) in males.

The need to consider an asthma-chronic obstructive pulmonary disease (COPD) overlap (ACO) and exacerbationprone asthma as distinct phenotypes has also been emphasised [24].

\section{Defining Asthma Endotypes}

Indisputable cluster analysis is useful for identifying more homogeneous sub-phenotypes of asthma, but unfortunately the identified sub-phenotypes are often not associated with defined pathophysiological pathways or an effective response to treatment [25].

Therefore, the currently emerging opinion is that it is essential to identify asthma endotypes (a subgrouping of disease associated with distinct functional or pathologic mechanisms) [25]. Adding a genetic or blood biomarker, which is not required to define a phenotype but is essential in endotyping the patient [17], will help to explain the relationship between phenotype and endotype and understand how cellular and molecular pathways modulate the characteristics of asthma. This is critical for achieving a real improvement in the assessment, treatment and outcomes of asthmaimprovement that will be possible to attain by applying a personalized accurate approach [25].

According to the PRACTALL (PRACtical ALLergy) document, which is an initiative of the European Academy of Allergy and Clinical Immunology and the American Academy of Allergy, Asthma, and Immunology that aims to standardize the European and American approaches to best allergy practice and science, in endotyping a patient, it is first of all necessary to verify that the diagnosis of asthma is correct and co-morbidities are adequately treated [16]. Thereafter phenotyping of the patient will occur based on the visible properties, and then endotyping.

It is increasingly evident that there are numerous endotypes that are fundamentally different from each other. Awareness of these differences is completely changing the concept of asthma, which is now gradually being considered as a syndrome composed of different endotypes [2].

The differences between asthma endotypes identified so far indicate that the main pathophysiological mechanisms causing these endotypes are fundamentally different. Therefore, it is incontestably necessary to characterise the endotype of patients not only to predict the risk, especially in those with severe and uncontrolled disease, but also to ensure the optimal response to treatment [25]. In fact, characterisation of endotypes allows us to appreciate and determine which specific targeted (or even non-targeted) immune therapies are more effective in relation to certain biologic characteristics [17]. It has been argued that allergic asthma is a distinct pheno-/endotype, despite the overlap with other pheno-/endotypes of eosinophilic and Type-2 high asthma [26].

Recent advances are accelerating the shift from phenotyping towards endotyping [17]. Moving from clusters of clinical and molecular phenotypes to endotypes can explore biological pathways in asthma [27]. Identification of any specific single pathway could help us improve our understanding of the drivers behind a person's asthma, and also discover and validate prognostic biomarkers related to disease severity and risk prediction (including the risk of developing asthma) that support new opportunities for primary and secondary asthma prevention [16]. It has been suggested that identification of disease mechanisms might be possible using statistical analysis of different levels of '-omic' data such as transcriptomics or proteomics [27].

The PRACTALL document confirmed that there are two major asthma types, the Type-2 immune response-driven (Type-2 high) asthma, and the non-Type-2 immune response (Type-2 low) asthma [16] (Fig. 1). The first type includes 
three main phenotypes: eosinophilic inflammation, allergic sensitisation-as depicted by the presence of antigen-specific immunoglobulin (Ig)E, and airway hyperresponsiveness (AHR) and remodelling. Furthermore, the PRACTALL document recognises several endotypes characterised and defined as an increase of $\mathrm{T}_{\mathrm{H}} 2$ cytokines, mainly of interleukin (IL)-4, IL-5, IL-13, IL-33 and thymic stromal lymphopoietin (TSLP), likely derived from adaptive (mainly $\mathrm{T}_{\mathrm{H}} 2$ lymphocytes) and/or innate (mainly innate lymphoid cells Type-2 (ILC2)) immune cells resulting in eosinophilic airway infiltration.

Endotype-driven asthma management targets most of the molecular pathways involved in Type-2 high asthma. There are now five approved biologic agents available that can target Type-2 high asthma (Table 1): omalizumab, a recombinant humanized monoclonal antibody $(\mathrm{mAb})$ that selectively binds circulating IgE in the blood and interstitial space; mepolizumab and reslizumab, which are mAbs directed against IL-5; benralizumab, a mAb directed against the $\alpha$ subunit of the IL-5 receptor; and dupilumab, which is an $\mathrm{mAb}$ directed against the IL-4 receptor $\alpha$, therefore targeting both IL-4 and IL-13 pathways. The available evidence indicates that omalizumab should be considered the firstchoice biological treatment for patients with allergic noneosinophilic severe asthma, high levels of blood $\mathrm{IgE}$, and at least a sensitisation to a perennial allergen [28]. Conversely, for patients with eosinophilic non-allergic severe asthma, it is reasonable to add an anti-IL-5 biological agent to the standard therapy. Anti-IL-4Ra is the treatment for severe eosinophilic Type-2 high asthma or patients requiring maintenance oral corticosteroids.

Type-2 low asthma includes three main phenotypes based on the inflammation pattern (neutrophilic or paucigranulocytic) and the presence of AHR and remodelling. It has been pointed out that endotypes of non-Type-2 asthma can further be classified according to the nature of underlying airway inflammation, as characterized by sputum cytometry, and AHR [29]. However, to date, no endotype-driven interventions have been shown to be effective for the Type- 2 endotype, although molecular pathways are under investigation in the hope of providing an endotype-driven approach [29]. The most commonly used therapies for non-Type- 2 asthma, tiotropium and macrolides, are prescribed on the basis of presumed efficacy and are not explicitly approved (or even studied) for this indication [15].

Recently, it has also been pointed out that the exposome could have a role in driving the disease phenotype and endotype [30]. The exposome considers exposures such as lifestyle factors (diet, smoking, etc.), air pollution, chemical exposures and many others an individual may have experienced in a lifetime and how those exposures relate to health. However, it is still unclear how this impacts the field of respiratory care [30].

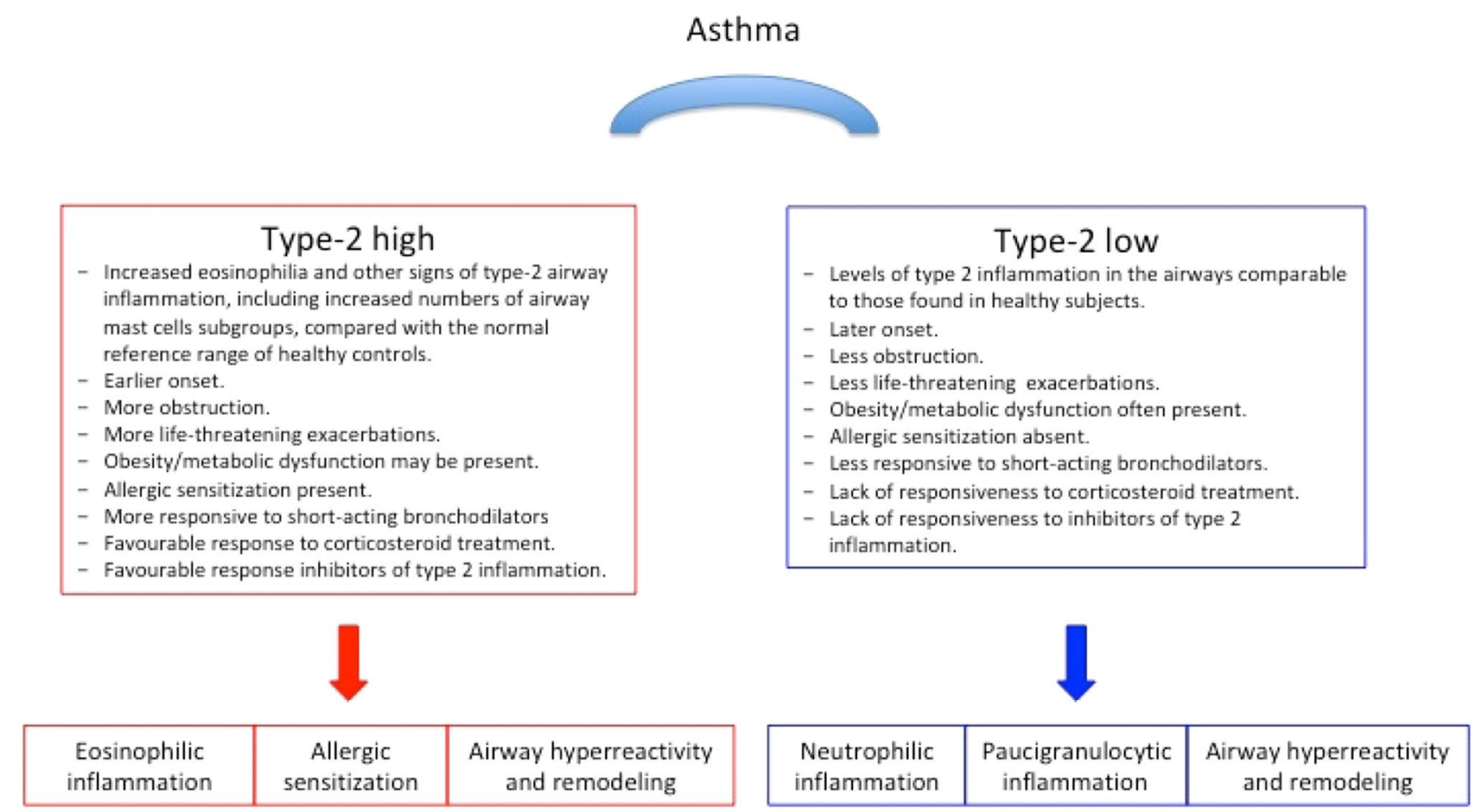

Fig. 1 Main pheno-/endotypes of asthma. From Fitzpatrick et al. [15] and Muraro et al. [16] (modified) 


\section{Treatable Traits in Asthma}

In order to overcome all problems related to the identification and validation of asthma phenotypes and endotypes, it has been proposed to use a precision-medicine strategy for the management of patients with airways disease that is free from the traditional diagnostic labels and is instead based on identifying 'treatable traits' in each patient [31]. This approach fully embraces the concept of precision medicine, which goes against the current 'one-size-fits-all' approach to asthma management [11]. It consists of the deconstruction of obstructive airways disease, including asthma, into its component parts to accurately identify and then target/treat the relevant traits in an individual [24].

The 'treatable' traits can be identified on a 'phenotypic' basis, i.e., by probabilistic evidence founded on positive and negative predictive values or, preferably, based on a deep understanding of the critical causal pathways, i.e. true 'endotypes'. Clinical phenotyping should lead to the identification of important treatable traits, which are clinical features that in any case characterise several clinical phenotypes [27]. This finding assumes that the same causal pathway can drive multiple treatable traits. However, a trait can be said to be treatable only when evidence-based treatments already exist to control that specific trait, which is usually not always the case. The whole process is then complicated by the frequent possibility that a given patient can present multiple treatable traits, which indicates that the presence of a trait does not exclude that of others [32].

Careful attention must also be paid to extrapulmonary comorbidities, which are important treatable traits to address in all patients with asthma, but particularly in patients with difficult asthma [33]. A stratified diagnostic approach seems to be of help in the search for these co-morbidities.

Candidate treatable traits must be clinically relevant, identifiable, measurable and treatable [26]. Multidimensional assessment enables detection of treatable traits and identifies a significant trait burden in severe asthma [34]. In particular, it accounts for asthma heterogeneity, which, as already mentioned, stems from several underlying phenotypes that can be impacted by several co-morbidities and risk factors [35].

Applying a multidimensional assessment of treatable traits in 140 patients with severe asthma, McDonald and colleagues identified $10.44 \pm 3.03$ traits per individual, comprising $3.01 \pm 1.54$ pulmonary, $4.85 \pm 1.86$ extrapulmonary and $2.58 \pm 1.31$ behavioural/risk factors [36]. The number of traits was significantly associated with health status (SGRQ).

Twenty-three treatable traits were identified in the U-BIOPRED (Unbiased Biomarkers for the Prediction of Respiratory Disease Outcomes) severe asthma cohort [37]. Seven out of the ten most prevalent traits in severe asthma (exercise-induced respiratory symptoms, cough, fixed airflow limitation, bronchodilator reversibility, bronchitis,

Table 1 Approved biologic agents available that can target Type-2 high asthma

\begin{tabular}{|c|c|c|c|c|}
\hline Biologic agent & Antibody type & Mechanism of action & Potential biomarkers & Indication \\
\hline Omalizumab & Humanised, IgG1 & $\begin{array}{l}\text { Binds circulating IgE in the blood } \\
\text { and interstitial space blocking its } \\
\text { interaction with FceRI }\end{array}$ & $\begin{array}{l}\text { Blood eosinophils } \uparrow \text { FeNO } \uparrow \\
\text { Blood periostin } \uparrow\end{array}$ & $\begin{array}{l}\text { First-choice biological treatment for } \\
\text { patients with allergic } \\
\text { non-eosinophilic severe asthma, } \\
\text { high levels of blood IgE and at } \\
\text { least a sensitisation to a perennial } \\
\text { allergen }\end{array}$ \\
\hline Mepolizumab & Humanised, IgG1/א & Directed against free IL-5 & $\begin{array}{l}\text { Eosinophils in sputum } \uparrow \\
\text { Blood eosinophils } \uparrow\end{array}$ & $\begin{array}{l}\text { Add-on treatment for patients with } \\
\text { severe eosinophilic asthma that } \\
\text { remain uncontrolled despite GINA } \\
\text { step } 4 \text { therapy }\end{array}$ \\
\hline Reslizumab & Humanised, IgG4 & Directed against free IL-5 & $\begin{array}{l}\text { Eosinophils in sputum } \uparrow \\
\text { Blood eosinophils } \uparrow\end{array}$ & $\begin{array}{l}\text { Add-on treatment for patients with } \\
\text { severe eosinophilic asthma that } \\
\text { remain uncontrolled despite GINA } \\
\text { step } 4 \text { therapy }\end{array}$ \\
\hline Benralizumab & Humanised, IgG1/א & $\begin{array}{l}\text { Directed against the } \alpha \text { subunit of the } \\
\text { IL-5 receptor (CD125) }\end{array}$ & $\begin{array}{l}\text { Eosinophils in sputum } \uparrow \\
\text { Blood eosinophils } \uparrow\end{array}$ & $\begin{array}{l}\text { Add-on treatment for inadequately } \\
\text { controlled severe eosinophilic } \\
\text { asthma }\end{array}$ \\
\hline Dupilumab & Fully human, IgG4 & $\begin{array}{l}\text { Directed against IL- } 4 \text { receptor } \alpha \\
\text { inhibiting the signalling of IL-4 } \\
\text { and IL-13 }\end{array}$ & $\begin{array}{l}\text { Blood eosinophils } \uparrow \\
\text { FeNO } \uparrow\end{array}$ & $\begin{array}{l}\text { Treatment for severe eosinophilic } \\
\text { Type- } 2 \text { high asthma or patients } \\
\text { requiring maintenance oral } \\
\text { corticosteroids }\end{array}$ \\
\hline
\end{tabular}

$I g$ immunoglobulin, $I L$ interleukin, $F e N O$ fractional exhaled nitric oxide, GINA Global Initiative for Asthma, $\uparrow$ indicates increase 
Type-2 inflammation and neutrophilic inflammation) were classified as treatable pulmonary traits (Fig. 2). Their prevalence in severe asthma compared to mild/moderate nonsmoking asthma was $84 \%$ versus $64 \%, 64 \%$ versus $22 \%$, $59 \%$ versus $20 \%, 59 \%$ versus $39 \%, 51 \%$ versus $18 \%, 44 \%$ versus $34 \%$ and $40 \%$ versus $30 \%$, respectively, while the most prevalent extrapulmonary traits were atopy $(71 \%$ vs. $90 \%$ ), rhinosinusitis ( $48 \%$ vs. $40 \%$ ), obesity ( $39 \%$ vs. $18 \%$ ), gastro-oesophageal reflux (36\% vs. $11 \%$ ), and obstructive sleep apnoea ( $26 \%$ vs. $11 \%)$.

In the Australasian SAWD (Severe Asthma WebBased Database), 24 treatable traits were identified in three domains: pulmonary, extrapulmonary and behavioural/risk factors [38]. The more prevalent traits found in severe asthma compared to non-severe asthma were: a non-completely reversible limitation to airway flow $(58.3 \%$ vs. 39.1$)$; being prone to exacerbation ( $51.4 \%$ vs. $5.9 \%)$; obesity ( $49.4 \%$ vs. $26.5 \%)$; gastro-oesophageal reflux disease $(49.1 \%$ vs. $36.3 \%)$; systemic inflammation $(41.0 \%$ vs. $13.2 \%$ ); Aspergillus sensitisation (38.7\% vs. $19.3 \%$ ); inhaler device polypharmacy ( $29.2 \%$ vs. $17.6 \%)$; depression (24.9\% vs. $9.1 \%)$; neutrophilic airway inflammation $(23.3 \%$ vs. $3.7 \%)$; obstructive sleep apnoea (OSA) $(17.3 \%$ vs. $6.9 \%)$; and vocal cord dysfunction (VCD) $(6.7 \%$ vs. $1.0 \%)$. Ten traits predicted exacerbation risk, with being prone to exacerbations prior to study entry, number of follow-up visits completed, inhaler device polypharmacy, depression, VCD and OSA the strongest predictors.
Although targeting treatments to these traits leads to significant improvements in health status, asthma control and asthma attacks [36], there is still no full understanding of the mechanisms underlying these treatable traits that allows us to direct any targeted therapies against any particular treatable trait; this is also because the definition of a treatable trait is too imprecise [27].

\section{Precision Medicine in Asthma}

The treatable traits approach is an interesting attempt to introduce precision medicine in asthma, and the identification of treatable traits could help optimize the treatment in this heterogeneous disorder [39]. However, it is still a rather unsophisticated approach. It is widely agreed that additional knowledge about the mechanisms underlying these treatable traits is needed to correctly guide targeted treatment based on identified traits in the individual patient. A systems biology approach could be extremely useful in paving the way for identifying mechanisms for treatable traits [27]. In effect, in-depth identification of underlying molecular pathways to guide targeted treatment in individual patients is already in progress [39], although it has not yet provided any decisive information.

Unfortunately, it is still true that most guideline-associated definitions include inflammation as a key characteristic,

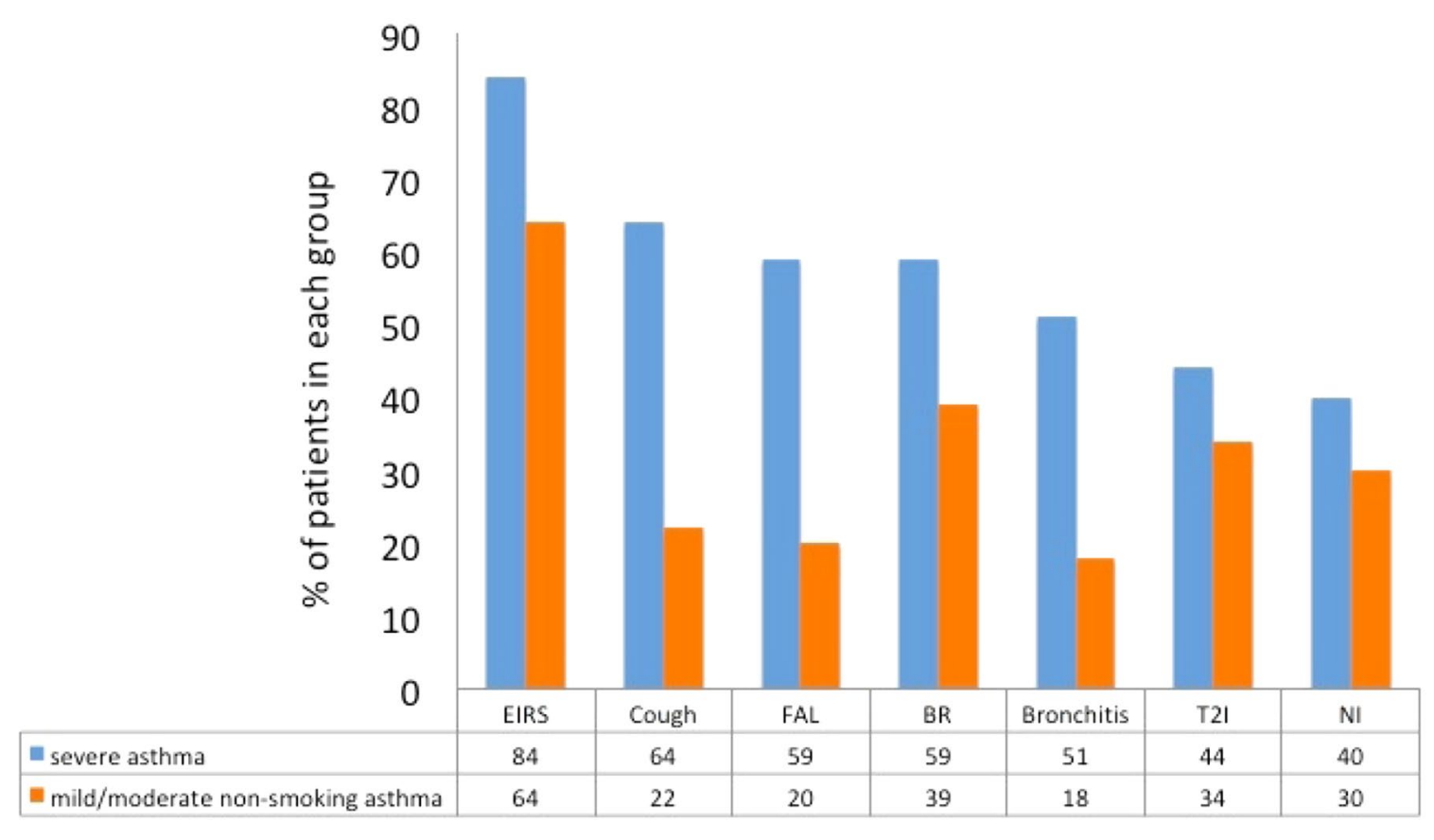

Fig. 2 Pulmonary treatable traits identified in the European Unbiased Biomarkers for the Prediction of Respiratory Diseases Outcomes (U-BIOPRED). EIRS exercise-induced respiratory symptoms, $F A L$ fixed airflow limitation, $B R$ bronchodilator reversibility, T2I Type-2 inflammation, $N I$ neutrophilic inflammation. From Simpson et al. [37] (modified) 
but without indication of specific inflammatory biomarkers [40], while it is widely accepted that the use of biomarkers reflecting activation of these different pathways is a high priority in applying precision medicine in asthma [41]. Undoubtedly, there is a need for reliable biomarkers that allow the identification of activated inflammatory pathways and treatment strategies in asthma patients. These biomarkers should be easy to measure, reproducible, and provide additive clinical value in terms of diagnosis, prognosis, monitoring and/or guiding therapy [36]. The need is even more urgent for non-Type-2 asthma.

FeNO and blood eosinophils are the only asthma biomarkers in clinical use in which elevated levels reflect ongoing Type-2 inflammation [39]. FeNO is more indicative of IL-4/IL-13 pathway activation, whereas blood eosinophils are more indicative of IL-5 pathway activation, although it is well known that IL-13 signalling can increase serum eosinophils and FeNO [39]. However, because blood eosinophil cell counts are relatively easy to obtain and have demonstrated clinical efficacy in distinguishing responders from non-responders in Type- 2 biologic trials, they remain the most established test to distinguish Type-2 high from Type-2 low asthma [42]. FeNO may provide added benefit when compared with blood eosinophil measures or in instances when the eosinophil count is low but suspicion for Type 2 inflammation is high. Conversely, the overlap between highIgE and Type-2 high asthma remains unclear. In fact, a large database study identified a considerable group of patients with severe asthma with low IgE/high blood eosinophils [43]. Much attention has also been paid to periostin, and serpin $\beta 2$ in the serum, but it is currently thought that they, similar to IgE levels, do not help to identify the Type- 2 high endotype [44].

Consequently, there is an urgent need to find better indicators for asthma pheno-/endotypes and to re-consider subgrouping of asthma [45]. Nevertheless, it has been opportunely highlighted that the absence or presence of these biomarkers can help identify individual patients who are likely non-responders or, instead, respond better to current therapies, including not only ICSs but also some biological and small molecules [36].

FeNO measurement, for example, shows promise in predicting potential response to ICS treatment, and, in combination with blood eosinophil count, to assess future risk [46]. High levels of blood eosinophils, FeNO or blood periostin are more likely to derive greater benefit from therapy with omalizumab, the dosage and frequency of administration of which, however, depend on serum total $\mathrm{IgE}$ level (IU/mL) measured before the start of treatment and body weight (in kilograms) [47]. Routine measurements of free IgE in serum can identify patients not responding to omalizumab therapy [48]. A higher baseline blood eosinophil count predicts responsiveness to anti-IL5 mAbs independently of serum
IgE concentrations [49]. Patients are more likely to respond to anti-IL- 5 treatment if they have $>3 \%$ of eosinophils in sputum, or $\geq 500$ cells $/ \mu \mathrm{L}$ blood eosinophils [50]. Subjects with higher baseline eosinophilia and FeNO levels showed greater benefits from treatment with dupilumab [51]. The presence of sputum neutrophilia is a predictive biomarker for macrolides in refractory asthma. Patients with sputum neutrophilia of $>61 \%$ seem to have greater improvements in quality-of-life scores compared with those without sputum neutrophilia [52]. This implies the need for more selective biomarkers (or panels) in order to identify the right biologic therapy for individual patients, in a more personalised and precise medicine approach to the disease treatment, and also to avoid potential side effects associated with unnecessary long-term use of these therapies [36]. The eosinophil depletion induced by anti-IL5 agents can potentially reduce the protective effects of eosinophils on neoplasia, infection and autoimmunity in humans, and consequently compromises safety [53]. The determination of Type- 2 biomarkers to use ICSs correctly appears to be much more urgent. The British Thoracic Society/Scottish Intercollegiate Guidelines Network guidelines recommend ICS therapy in all patients once the diagnosis of asthma is confirmed [54], but the available evidence suggests that adults with asthma who are prescribed medium or high ICS doses are at risk of clinically important systemic side effects [55]. The presence of Type-2 inflammation requires the use of ICSs. Thus, the determination of Type- 2 biomarker status is fundamental because it has implications in terms of deciding whether to treat the patient with an ICS and determining whether he/she requires relatively higher doses of ICS [56].

The development of 'multi-omic' platforms, capable of measuring hundreds to even millions of analytes, typically of a specific type, on a single specimen, has opened the floodgates for molecular phenotyping by significantly increasing the complexity and granularity of molecular data [40]. In fact, the improvement in big data management obtained from '-omic' sciences (genomics, transcriptomics, proteomics, metabolomics, lipidomics, glyconomics, epigenomics) is greatly increasing the knowledge concerning asthma [57]. Integrative '-omics' (multi-omics) is combining the information of multiple '-omics' layers in order to obtain more insight into the asthma disease process [45]. However, despite the growing importance of '-omics' in the asthma research field, there are various methodological, practical and ethical challenges that currently hinder appropriate analysis, interpretation and clinical application of '-omic' data [57].

A clever and accurate application of the '-omic' sciences can allow the replacement of phenotypes with the currently emerging concept of 'treatable mechanisms'. Based on '-omic' sciences, numerous up- or downregulated pathways have been or are being identified. Such pathways can be 
understood as potential robust biomarkers with the needed accuracy for precision medicine that, to be successful, must be predictive, preventive, personalised and participative [57]. These biomarkers will surely help to better model asthma and provide 'care and cure' at an individual patient level, and thus move from dissociated fingerprints to integrative handprints [58].

It has long been accepted that the heterogeneity and complexity of the asthma syndrome require a different approach to its management that goes beyond the use of clinical characteristics and physiological and inflammatory data, which are now clearly no longer sufficient [59]. Unbiased clustering and the application of '-omic' data will likely allow phenotyping asthma based on the mechanisms that drive the pathologic process in each phenotype. The subsequent use of a composite set of biomarkers will then help to define and classify the endotypes. When all this is possible in routine practice, a personalised medicine for asthma will surely develop that will allow not only more precise treatment but also help to identify new traits/targets and therefore new treatments for each defined endotype.

Future '-omic' studies should also evaluate the pathogenic mechanisms in the elderly and in severe adult-onset asthma, considering the possible sex-specific differences, as this approach is likely to be the only one that can allow identification of target therapies for these populations with a poorly controlled disease.

In the meantime, pending the availability of such data, it is certainly wrong to assume that the current inability to correctly treat a trait is an absolute barrier to its recognition. Rather, traits that are strongly associated with important outcomes but are not yet curable should be considered as important opportunities for the development of new therapies [32], although some traits may require considerable human and economic resources for their investigation.

In any case, it is high time that personalised medicine be applied to the whole spectrum of asthma, not just to severe asthma. However, there is still no consensus on what traits should form the basis of a multidimensional assessment, what traits treatment should target, and what model of care should be used to implement the precision medicine approach in asthma.

\section{Declarations}

Funding Open Access funding provided by Università degli Studi di Roma Tor Vergata.

Conflict of interest Mario Cazzola, Josuel Ora, Francesco Cavalli, Paola Rogliani and Maria Gabriella Matera have no relevant affiliations or financial involvement with any organisation or entity with a financial interest in, or financial conflict with, the subject matter or materials discussed in the manuscript, including employment, consultan- cies, honoraria, stock ownership or options, expert testimony, grants or patents received or pending, or royalties.

Ethics approval Not applicable.

Consent to participate Not applicable.

Consent for publication Not applicable.

Availability of data and material Not applicable.

Code availability Not applicable.

Authors' contributions All authors contributed to the study conception. The first draft of the manuscript was written by Mario Cazzola and Maria Gabriella Matera and all authors commented on previous versions of the manuscript. All authors read and approved the final manuscript.

Open Access This article is licensed under a Creative Commons Attribution-NonCommercial 4.0 International License, which permits any non-commercial use, sharing, adaptation, distribution and reproduction in any medium or format, as long as you give appropriate credit to the original author(s) and the source, provide a link to the Creative Commons licence, and indicate if changes were made. The images or other third party material in this article are included in the article's Creative Commons licence, unless indicated otherwise in a credit line to the material. If material is not included in the article's Creative Commons licence and your intended use is not permitted by statutory regulation or exceeds the permitted use, you will need to obtain permission directly from the copyright holder. To view a copy of this licence, visit http://creativecommons.org/licenses/by-nc/4.0/.

\section{References}

1. National Heart, Lung, and Blood Institute, National Institutes of Health, US Department of Health and Human Services. Expert panel report 3: guidelines for the diagnosis and management of asthma. Section 2, p. 1. 2007. http://www.nhlbi.nih.gov/guidelines /asthma/asthgdln.ht. Accessed 21 Oct 2020.

2. Lötvall J, Akdis CA, Bacharier LB, Bjermer L, Casale TB, Custovic A, et al. Asthma endotypes: a new approach to classification of disease entities within the asthma syndrome. J Allergy Clin Immunol. 2011;127(2):355-60.

3. Wenzel SE. Asthma: defining of the persistent adult phenotypes. Lancet. 2006;368(9537):804-13.

4. Wenzel SE. Asthma phenotypes: the evolution from clinical to molecular approaches. Nat Med. 2012;18(5):716-25.

5. Global initiative for asthma. 2020 GINA report, global strategy for asthma management and prevention. https://ginasthma.org/wpcontent/uploads/2020/04/GINA-2020-full-report_-final-_wms. pdf. Accessed 21 Oct 2020.

6. Agache I, Akdis CA. Precision medicine and phenotypes, endotypes, genotypes, regiotypes, and theratypes of allergic diseases. J Clin Investig. 2019;129(4):1493-503.

7. Santus P, Saad M, Damiani G, Patella V, Radovanovic D. Current and future targeted therapies for severe asthma: managing treatment with biologics based on phenotypes and biomarkers. Pharmacol Res. 2019;146:104296.

8. Chung KF. Defining phenotypes in asthma: a step towards personalised medicine. Drugs. 2014;74(7):719-28. 
9. President's Council of Advisors on Science and Technology. Priorities for personalized medicine. 2008. https://www.whitehouse .gov/files/documents/ostp/PCAST/pcast_report_v2.pdf. Accessed 21 Oct 2020

10. Mirnezami R, Nicholson J, Darzi A. Preparing for precision medicine. N Engl J Med. 2012;366(6):489-91.

11. Pavord ID, Beasley R, Agusti A, Anderson GP, Bel E, Brusselle $\mathrm{G}$, et al. After asthma: redefining airways diseases. Lancet. 2018;391(10118):350-400.

12. Fahy JV. Type 2 inflammation in asthma-present in most, absent in many. Nat Rev Immunol. 2015;15(1):57-65.

13. Oishi K, Hirano T, Matsuda K, Hamada K, Uehara S, Suetake R, et al. The prevalence and clinical characteristics of T2-high and T2-low endotype of severe uncontrolled asthma distinguished by combining popular type-2 biomarkers (abstract). Eur Respir J. 2019;54:PA2749.

14. Jackson D, Aljamil N, Roxas C, Fernandes M, Green L, Kavanagh J, et al. P48 The 'T2-low' asthma phenotype: could it just be T2-high asthma treated with corticosteroids? (abstract). Thorax. 2018;73:A124-5.

15. Fitzpatrick AM, Chipps BE, Holguin F, Woodruff PG. T2-"low" asthma: overview and management strategies. J Allergy Clin Immunol Pract. 2020;8(2):452-63.

16. Muraro A, Lemanske RF Jr, Hellings PW, Akdis CA, Bieber $\mathrm{T}$, Casale TB, et al. Precision medicine in patients with allergic diseases: airway diseases and atopic dermatitis-PRACTALL document of the European Academy of Allergy and Clinical Immunology and the American Academy of Allergy, Asthma \& Immunology. J Allergy Clin Immunol. 2016;137(5):1347-58.

17. Wenzel SE. Complex phenotypes in asthma: current definitions. Pulm Pharmacol Ther. 2013;26(6):710-5.

18. Lefaudeux D, De Meulder B, Loza MJ, Peffer N, Rowe A, Baribaud F, et al. U-BIOPRED clinical adult asthma clusters linked to a subset of sputum omics. J Allergy Clin Immunol. 2017;139(6):1797-807.

19. Loza MJ, Djukanovic R, Chung KF, Horowitz D, Ma K, Branigan $\mathrm{P}$, et al. Validated and longitudinally stable asthma phenotypes based on cluster analysis of the ADEPT study. Respir Res. 2016;17(1):165.

20. Kaur R, Chupp G. Phenotypes and endotypes of adult asthma: Moving toward precision medicine. J Allergy Clin Immunol. 2019;144(1):1-12.

21. Chaudhuri R, McSharry C, Heaney LG, Niven R, Brightling CE, Menzies-Gow AN, et al. Effects of older age and age of asthma onset on clinical and inflammatory variables in severe refractory asthma. Respir Med. 2016;118:46-52.

22. Principe S, Benfante A, Calzetta L, Rogliani P, Scichilone N. Age does not affect the efficacy of anti-IL-5/IL-5R in severe asthmatics. World Allergy Organ J. 2019;12(11):100081.

23. Hsiao HP, Lin MC, Wu CC, Wang CC, Wang TN. Sex-specific asthma phenotypes, inflammatory patterns, and asthma control in a cluster analysis. J Allergy Clin Immunol Pract. 2019;7(2):556-67.

24. Fuhlbrigge AL, Castro M. Precision medicine in asthma - Using phenotypes to understand endotypes that lead us to new therapeutic options. J Allergy Clin Immunol Pract. 2020;8(2):496-7.

25. Li X. Hot Topic: Precision medicine for asthma-has the time come? Curr Allergy Asthma Rep. 2019;19(10):45.

26. Akar-Ghibril N, Casale T, Custovic A, Phipatanakul W. Allergic endotypes and phenotypes of asthma. J Allergy Clin Immunol Pract. 2020;8(2):429-40

27. Chung KF, Adcock IM. Precision medicine for the discovery of treatable mechanisms in severe asthma. Allergy. 2019;74(9):1649-59.
28. Rogliani P, Calzetta L, Matera MG, Laitano R, Ritondo BL, Hanania NA, et al. Severe asthma and biological therapy: when, which, and for whom. Pulm Ther. 2020;6(1):47-66.

29. Sze E, Bhalla A, Nair P. Mechanisms and therapeutic strategies for non-T2 asthma. Allergy. 2020;75(2):311-25.

30. Agache I, Miller R, Gern JE, Hellings PW, Jutel M, Muraro A, et al. Emerging concepts and challenges in implementing the exposome paradigm in allergic diseases and asthma: a Practall document. Allergy. 2019;74(3):449-63.

31. Agusti A, Bel E, Thomas M, Vogelmeier C, Brusselle G, Holgate $\mathrm{S}$, et al. Treatable traits: toward precision medicine of chronic airway diseases. Eur Respir J. 2016;47(2):410-9.

32. McDonald VM, Fingleton J, Agusti A, Hiles SA, Clark VL, Holland AE, et al. Treatable traits: a new paradigm for 21st century management of chronic airway diseases: Treatable Traits Down Under International Workshop report. Eur Respir J. 2019;53(5):1802058.

33. Tay TR, Hew M. Comorbid, "treatable traits" in difficult asthma: current evidence and clinical evaluation. Allergy. 2018;73(7):1369-82.

34. Hiles SA, Gibson PG, Agusti A, McDonald VM. Treatable traits that predict health status and treatment response in airway disease. J Allergy Clin Immunol Pract. 2020. https://doi.org/10.1016/j. jaip.2020.09.046.

35. Clark VL, Gibson PG, Genn G, Hiles SA, Pavord ID, McDonald VM. Multidimensional assessment of severe asthma: a systematic review and meta-analysis. Respirology. 2017;22(7):1262-75.

36. McDonald VM, Clark VL, Cordova-Rivera L, Wark PAB, Baines KJ, Gibson PG. Targeting treatable traits in severe asthma: a randomised controlled trial. Eur Respir J. 2020;55(3):1901509.

37. Simpson AJ, Hekking PP, Shaw DE, Fleming LJ, Roberts G, Riley JH, et al. Treatable traits in the European U-BIOPRED adult asthma cohorts. Allergy. 2019;74(2):406-11.

38. McDonald VM, Hiles SA, Godbout K, Harvey ES, Marks GB, Hew M, et al. Treatable traits can be identified in a severe asthma registry and predict future exacerbations. Respirology. 2019;24(1):37-47.

39. Ulrik CS, Vijverberg S, Hanania NA, Diamant Z. Precision medicine and treatable traits in chronic airway diseases-where do we stand? Curr Opin Pulm Med. 2020;26(1):33-9.

40. Ray A, Camiolo M, Fitzpatrick A, Gauthier M, Wenzel SE. Are we meeting the promise of endotypes and precision medicine in asthma? Physiol Rev. 2020;100(3):983-1017.

41. Mogensen I, James A, Malinovschi A. Systemic and breath biomarkers for asthma: an update. Curr Opin Allergy Clin Immunol. 2020;20(1):71-9.

42. Coverstone AM, Seibold MA, Peters MC. Diagnosis and management of T2-high asthma. J Allergy Clin Immunol Pract. 2020;8(2):442-50.

43. Haughney J, Morice A, Blyth KG, Lee AJ, Coutts A, McKnight E, et al. A retrospective cohort study in severe asthma describing commonly measured biomarkers: eosinophil count and IgE levels. Respir Med. 2018;134:117-23.

44. Pavlidis S, Takahashi K, Kwong FNK, Xie J, Hoda U, Sun $\mathrm{K}$, et al. "T2-high" in severe asthma related to blood eosinophil, exhaled nitric oxide and serum periostin. Eur Respir J. 2019;53(1):1800938.

45. Roth M, Stolz D. Biomarkers and personalised medicine for asthma. Eur Respir J. 2019;53(1):1802094.

46. Alving K, Diamant Z, Lucas S, Magnussen H, Pavord ID, Piacentini G, et al. Point-of-care biomarkers in asthma management: time to move forward. Allergy. 2020;75(4):995-7.

47. Hanania NA, Wenzel S, Rosén K, Hsieh HJ, Mosesova S, Choy DF, et al. Exploring the effects of omalizumab in allergic asthma: an analysis of biomarkers in the EXTRA study. Am J Respir Crit Care Med. 2013;187(8):804-11. 
48. Korn S, Haasler I, Fliedner F, Becher G, Strohner P, Staatz A, et al. Monitoring free serum IgE in severe asthma patients treated with omalizumab. Respir Med. 2012;106(11):1494-500.

49. Calzetta L, Matera MG, Coppola A, Rogliani P. Prospects for severe asthma treatment. Curr Opin Pharmacol. 2020;56:52-60.

50. Diamant Z, Vijverberg S, Alving K, Bakirtas A, Bjermer L, Custovic A, et al. Toward clinically applicable biomarkers for asthma: an EAACI position paper. Allergy. 2019;74(10):1835-51.

51. Castro M, Corren J, Pavord ID, Maspero J, Wenzel S, Rabe KF, et al. Dupilumab efficacy and safety in moderate-to-severe uncontrolled asthma. N Engl J Med. 2018;378(26):2486-96.

52. Simpson JL, Powell H, Boyle MJ, Scott RJ, Gibson PG. Clarithromycin targets neutrophilic airway inflammation in refractory asthma. Am J Respir Crit Care Med. 2008;177(2):148-55.

53. Bagnasco D, Ferrando M, Varricchi G, Puggioni F, Passalacqua G, Canonica GW. Anti-interleukin 5 (IL-5) and IL-5Ra biological drugs: efficacy, safety, and future perspectives in severe eosinophilic asthma. Front Med (Lausanne). 2017;4:135.

54. British Thoracic Society/Scottish Intercollegiate Guidelines Network. British guideline on the management of asthma: a national clinical guideline; 2016. http://www.brit-thoracic.org. uk. Accessed 5 Jan 2021.
55. Irwin RS, Richardson ND. Side effects with inhaled corticosteroids: the physician's perception. Chest. 2006;130(1 Suppl):41S-53S.

56. Beasley R, Harper J, Bird G, Maijers I, Weatherall M, Pavord ID. Inhaled corticosteroid therapy in adult asthma. Time for a new therapeutic dose terminology. Am J Respir Crit Care Med. 2019;199(12):1471-7.

57. Abdel-Aziz MI, Neerincx AH, Vijverberg SJ, Kraneveld AD, Maitland-van der Zee AH. Omics for the future in asthma. Semin Immunopathol. 2020;42(1):111-26.

58. Colas L, Hassoun D, Magnan A. Needs for systems approaches to better treat individuals with severe asthma: predicting phenotypes and responses to treatments. Front Med (Lausanne). 2020;7:98.

59. Chung KF. Personalised medicine in asthma: time for action. Eur Respir Rev. 2017;26(145):170064. 